\title{
EDITORIAL
}

\section{Spin-off Companies: A Great Challenge for the Brazilian Space Program}

\author{
Marcia Mantelli ${ }^{1}$
}

S pace science research and development is, by itself, a great challenge for research institutions throughout the world. This is especially true for developing countries such as Brazil, where the expenditures and number of institutions devoted to this field of study are limited. Additionally, because most funding for space research programs often originates from government sources, these are usually one of the first to be cut in times of economic distress. On the other hand, economy studies of the main space programs in the world show that the return on investment to the society in terms of new technologies greatly surpasses the research funds. Therefore, one of the greatest challenges for governments of developing countries with their own space programs is to create favorable conditions, so that the investments become profitable to the population, providing not only technological independence in the space field but also technological solutions to daily problems.

Development of the heat pipe technology is a good illustration of the aforementioned tradeoff. Heat pipes are highly efficient, low cost passive heat transfer devices, used for the thermal management of equipment and panels in satellites. The first registered patents are concerned with application of these devices in domestic and bakery applications, dating back to the middle of the $20^{\text {th }}$ century. Although very efficient, this technology was not largely employed until the Cold War, when the major countries intensified their space exploration programs, leading to the need for reliable space-applicable technologies. In case of Brazil, heat pipe research and development started at the Instituto Nacional de Pesquisas Espaciais (INPE) in the late 1980s to provide thermal solutions for satellites. At that time, several cooperation agreements among Brazilian institutions devoted to spacerelated activities; Instituto Nacional de Pesquisas Espaciais (INPE), Departamento de Ciência e Tecnologia Aeroespacial (DCTA), Instituto de Aeronáutica e Espaço (IAE), etc., and Brazilian Universities; Universidade Federal do Rio de Janeiro (UFRJ), Universidade Federal de Santa Catarina (UFSC), Universidade de São Paulo (USP), etc., were established, aiming to promote research and development of spacecraft subsystems (satellites and rockets) as well as of onboard equipment and software. This effort allowed fulfillment of the objectives of the Brazilian Space Program and created a highly qualified workforce at INPE and other involved institutions. In this context, INPE and UFSC established their first common heat pipe development project. In the 1990s, the Brazilian Space Agency (AEB) was established and new contracts were signed between $\mathrm{AEB}$ and Brazilian Universities, allowing the establishment of reference laboratories and development of several productive research groups in Brazil.

Although the Brazilian Space Program is still modest in comparison with other existing programs, several satellites have been successfully launched. For several years, programs

1.Professor at Department of Mechanical Engineering/Universidade Federal de Santa Catarina/Brazil, and coordinator of the research and development activities of the Laboratory of Heat Pipes. She was the first woman to graduate as a Mechanical Engineer from UNICAMP, Brazil in 1983 and received the degree of Master in Space Science by the Instituto Nacional de Pesquisas Espaciais, Brazil, where she worked from 1886 to 1999. She received her Ph.D. from the University of Waterloo, Canada in 1995. Approximately 50 research projects on the development of equipment for industry with heat pipes and thermosyphons were coordinated or are in progress, involving approximately 40 masters and doctoral students. She has published around 30 articles in specialized journals, and presented more than 100 conference articles. She also participates in editorial board and as a reviewer of several journals worldwide. She received, among others, the Claudia Award, Editora Abril, in the Sciences category in 2012. Email: marcia@labtucal.ufsc.br 
such as "Microgravity" and "Uniespaço", coordinated by AEB, have been providing space-related research opportunities for many different fields of science in Brazil. As a "side effect" of the Brazilian Space Program, many laboratories received a strong investment from the national industry, aiming the application of space technology to solve a large spectrum of practical problems. Additionally, in the last three decades, a highly qualified workforce in space-related subjects, mainly composed by engineers, was formed. Again, the heat pipe technology can be cited as an example, as this technology is being considered to be employed to thermal management in the petroleum, aeronautics, electronics and food industries, particularly when low cost and highly thermally efficient equipment are required.

As the industry becomes increasingly convinced of the applicability of novel space-related technological solutions, a new problem emerges: who will produce the equipment to fulfill the needs of this emergent market?
As a developing country, Brazil has a growing number of highly qualified well-trained people, but it does not have a strong tradition in starting-up new technological companies, although incubators have being active for at least two decades. Furthermore, some of the start-up companies have a short life. Thus, the start-up and growth of new strong technologybased companies is a great challenge for governments of developing countries such as Brazil.

In conclusion, the start-up of new technology-based companies is a "side effect" of space programs that should be taken into consideration when national space programs are being established. Considering the larger and recently established United States IT enterprises as an example, one can conclude that space program spin-off companies have large potential to be highly successful if their start-up process is well conducted. 